\title{
Can breast microbiota provide protective effects against cancer?
}

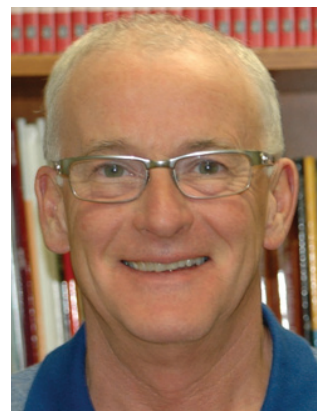

Gregor Reid*
“The breadth of bacterial species in milk has only recently been uncovered by molecular

First draft submitted: 6 July 2016; Accepted for publication: 8 July 2016;

Published online: 5 August 2016

It has long been known that human milk contains bacteria, and these help prime the newborn's immune system and ongoing organ development. The breadth of bacterial species in milk has only recently been uncovered by molecular techniques [1-4] . Perhaps not surprisingly, there are differences between samples, in terms of bacterial types and abundance, but whether this translates to functional differences in the health programming of the newborn remains to be studied.

Breastfeeding is promoted globally as a means to pass nutrients to the newborn, yet in many so-called developed countries, rates are plummeting. Ironically, breast cancer rates can be as low as 19 per 100,000 women in Eastern Africa to 90 in Western Europe [5], and there are no signs of it decreasing. Counter this with the fact that breastfeeding can reduce the risk of breast cancer [6]. Of the many issues raised by these statistics, several questions come to mind. What if a woman never lactates or breastfeeds, does she still have bacteria in her mammary gland? In addition to the obvious entrance of bacteria through the nipple, are there other ways in which such a wide range of bacteria reach the breast? Why do species like E. coli, Bacillus and Pseudomonas, found in nutrient-rich breast milk, not induce infection or cancer given that they have well-known properties to cause either? We hypothesized that the risk of cancer is associated with the microbiota composition and proliferation of beneficial lactic acid bacteria during lactation, reduces this risk by countering carcinogenic bacterial species.

Surgeons and pathologists at our site are hesitant to provide breast tissue from patients suspected of having cancer, in case the portion given for research has a different stage to the one examined for pathology. Nevertheless, bacteria were identified (and in some cases cultured) in tissue adjacent to tumors and the patterns differed from those of tissues recovered from healthy women $[7,8]$. Irish collaborators [7] and other researchers able to acquire tumor

\section{KEYWORDS}

- cancer $\bullet$ human breast $\bullet$ lactobacilli - microbiota

"The finding that recurrent use of antibiotics can promote some cancers, with sulfonamides associated with breast cancer, further implies a correlation between bacteria and cancer." 


\section{“For those already suffering from localized or metastatic cancer, the application of targeted probiotics and antibiotics} may contribute to disease management." samples [9], confirmed these findings, as did a recent study of bacteria in nipple aspirates [10]. Collectively, samples from 220 women were analyzed. Intriguingly, bacteria were detected in all areas of the fatty breast tissues examined, but in very low numbers. Attempts by us to visualize them with electron microscopy of tissues sections failed to find definitive colonies, demonstrating the sparsity. Presumably, host factors keep these organisms from proliferating, yet recovery by culture shows they are still metabolically active and the molecules they produce will soon be identified. Proving cause and effect of bacteria and cancer induction will be difficult, but studies showing they can damage DNA, produce carcinogens or secondary metabolites that are carcinogenic, induce tumorigenesis, and repress expression of tumor suppressors [4,11-12] supports the potential for them to be more than bystanders in carcinogenesis.

The finding that recurrent use of antibiotics can promote some cancers, with sulfonamides associated with breast cancer [13], further implies a correlation between bacteria and cancer. Notably, many women suffer from urinary tract infections that are treated with sulfonamides, and it is certainly possible that this disrupts the breast microbiota, as well as the intestinal and vaginal. Bacteriuria caused by $E$. coli causes inflammation and hypertension [14], and inflammation is associated with cancer. The fact that a urinary tract infection can induce hypertension illustrates that distant site effects can arise from bacteria invading tissues at another site. Once in the breast, Bacillus and E. coli can produce peptides that promote tumor cell invasion and angiogenesis [15], which can not only cause localized damage, but may even influence metastasis. The latter warrants further investigation and assessment of metastatic sites for the same organisms as in the breast tumor. If indeed bacteria take control of the tumor cells, and use of quorum sensing molecules provides an example of how bacteria can change the behavior and function of neutrophils, macrophages and epithelial cells [16], then this has major implications for cancer management. There is already evidence that bacteria in the gut can use neutrophils to reach the breast and promote mammary tumorogenesis [17]. In addition, a study has shown that ingested Lactobacillus plantarum NCIMB8826 could improve skin barrier integrity, and improve skin pathology [18], once again suggesting the potential to affect distant sites where breast cancer may have metastasized.
This distant site effect has direct implications for the breast microbiome, as shown by a study in which orally administered probiotic lactobacilli reached the breast and cured mastitis [19]. The is particularly intriguing as it provides a means to manipulate the breast microbiome and implant beneficial bacteria selected for their ability to counter the species or compounds associated with cancer. Such antagonism and ability to degrade some carcinogens [20] are hallmarks of some lactic acid bacteria, and may partly explain the reduced risk of cancer in breastfeeding women.

It may seem implausible to some that after a century of looking for dietary, genomic and environmental factors that cause breast cancer and treating with potent drugs, radiation, surgery and various high-tech approaches, tiny microorganisms are key participants. Admittedly, cause and effect is unproven, and nobody is suggesting that bacteria alone explain this complex condition. Likewise, nobody is proposing that probiotic lactobacilli can prevent or treat breast cancer, yet. But it behooves researchers and clinicians to consider the potential and implications of these discoveries, especially since some antibiotics are known to slow cancer progression. The future could well see nipple aspirates and needle biopsies being part of routine checks for aberrant microbiota and metabolomic patterns associated with increased risk of breast cancer. It may well see attempts to reset this microbiome through administration of beneficial bacteria via the nipple, gut and other sites. The first cohort to test such theories would be women at known risk of breast cancer, and who feel as did Angelina Jolie, that radical mastectomy is their only hope. For those already suffering from localized or metastatic cancer, the application of targeted probiotics and antibiotics may contribute to disease management.

The tendency has been to avoid use of products, such as probiotics, that interfere with the host's immune response, but given the toxicity of drug therapy and severity of side effect, it seems hard to imagine they would do more harm than good. Whatever studies are designed to test probiotic concepts for breast health, selection of the strains should be based upon safe use in humans, ability to support epithelial barrier function, expression of antipathogen characteristics, and the ability to either degrade or detoxify chemicals 
and metals known to increase the risk of cancer. Whether administered orally or at the nipple, a method should be devised to assess their survival and impact on the microbiota and metabolome of the breast. It is unlikely that changes to the microbiota will be permanent without lengthy application, based upon most probiotic studies at other sites, but drug therapy is already long term, so this approach is not unusual. Given the general health benefits conveyed by lactic acid bacteria, it behooves us to consider microbiome manipulation and use of beneficial microbes in cancer prevention and management.

\section{References}

Papers of special note have been highlighted as:

- of interest; $\bullet$ of considerable interest

1 Martín R, Langa S, Reviriego C et al. Human milk is a source of lactic acid bacteria for the infant gut. J. Pediatr. 143(6), 754-758 (2003).

2 Hunt KM, Foster JA, Forney LJ et al. Characterization of the diversity and temporal stability of bacterial communities in human milk. PLoS ONE 6(6), e21313 (2011).

- This paper uses high-throughput sequencing to show an extensive array of bacteria in human milk.

3 Bisanz JE, Enos MK, PrayGod G et al. Microbiota at multiple body sites during pregnancy in a rural Tanzanian population and effects of Moringa-supplemented probiotic yogurt. Appl. Environ. Microbiol. 81(15), 4965-4975 (2015).

4 Urbaniak C, Angelini M, Gloor GB, Reid G. Human milk microbiota profiles in relation to birthing method, gestation and infant gender. Microbiome 4, 1 (2016).

5 WHO. Breast cancer: prevention and control. www.who.int

6 Wynder EL. Identification of women at high risk for breast cancer. Cancer 24(6), 1235-1240 (1969).

7 Urbaniak C, Cummins J, Brackstone $\mathrm{M}$ et al. Microbiota of human breast tissue. Appl. Environ. Microbiol. 80 (10), 3007-3014 (2014).

- The first paper to show bacteria throughout the breast of women in Canada and Ireland. It has attracted a lot of interest from researchers and patients.

\section{Acknowledgements}

The author would like to thank C Urbaniak for her PhD work and her collaborators on this topic; the author appreciates the cooperation of all the patients studied.

\section{Financial \& competing interests disclosure}

The author has no relevant affliations or financial involvement with any organization or entity with a financial interest in or financial conflict with the subject matter or materials discussed in the manuscript. This includes employment, consultancies, honoraria, stock ownership or options, expert testimony, grants or patents received or pending, or royalties.

No writing assistance was utilized in the production of this manuscript.

8 Urbaniak C, Gloor GB, Brackstone M, Scott L, Tangney M, Reid G. The microbiota of breast tissue and its association with tumours. Appl. Environ.

Microbiol. pii:AEM.01235-16 (2016) (Epub ahead of print).

- Shows a distinct difference between cancer patients and controls, and shows that bacteria from patients could damage DNA while lactobacilli do not.

9 Xuan C, Shamonki JM, Chung A et al. Microbial dysbiosis is associated with human breast cancer. PLoS ONE 9(1), e83744 (2014).

10 Chan AA, Bashir M, Rivas MN et al. Characterization of the microbiome of nipple aspirate fluid of breast cancer survivors. Sci. Rep. 6, 28061 (2016).

-. This not only confirms the presence of a microbiota in the breast of cancer patients, but shows that a noninvasive aspirate has the potential to diagnosis dysbiosis.

11 Jin Y, Tang S, Li W et al. Hemolytic E. coli promotes colonic tumorigenesis in females. Cancer Res. 76(10), 2891-2900 (2016).

$12 \mathrm{Ou}$ J, Carbonero F, Zoetendal EG et al. Diet, microbiota, and microbial metabolites in colon cancer risk in rural Africans and African Americans. Am. J. Clin. Nutr. 98(1), 111-120 (2013).

13 Boursi B, Mamtani R, Haynes K, Yang YX. Recurrent antibiotic exposure may promote cancer formation - another step in understanding the role of the human microbiota? Eur. J. Cancer 51(17), 2655-2664 (2015).
14 Meiland R, Geerlings SE, Stolk RP et al. Escherichia coli bacteriuria in female adults is associated with the development of hypertension. Int. J. Infect. Dis. 14(4), e304-e307 (2010).

15 De Spiegeleer B, Verbeke F, D'Hondt $\mathrm{M}$ et al. The quorum sensing peptides PhrG, CSP and EDF promote angiogenesis and invasion of breast cancer cells in vitro. PLoS ONE 10(3), e0119471 (2015).

16 Holm A, Vikström E. Quorum sensing communication between bacteria and human cells: signals, targets, and functions. Front. Plant. Sci. 5, 309 (2014).

17 Lakritz JR, Poutahidis T, Mirabal S et al. Gut bacteria require neutrophils to promote mammary tumorigenesis. Oncotarget 6(11), 9387-9396 (2015).

18 Mariman R, Reefman E, Tielen F et al. Lactobacillus plantarum NCIMB8826 ameliorates inflammation of colon and skin in human APOC1 transgenic mice. Benef. Microbes 7(2), 215-225 (2016).

19 Arroyo R, Martín V, Maldonado A, Jiménez E, Fernández L, Rodríguez JM. Treatment of infectious mastitis during lactation: antibiotics versus oral administration of lactobacilli isolated from breast milk. Clin. Infect. Dis. 50(12), 1551-1558 (2010).

-. Proves that probiotic lactobacilli can reach the breast via the gut.

20 Nowak A, Kuberski S, Libudzisz Z. Probiotic lactic acid bacteria detoxify $N$ nitrosodimethylamine. Food Addit. Contam. Part A Chem. Anal. Control Expo. Risk Assess. 31(10), 1678-1687 (2014). 